\title{
Erector spinae plane block for postoperative analgesia in laparoscopic cholecystectomy: a case report
}

\author{
Dimosthenis Petsas \\ Valentini Pogiatzi' \\ Thanasis Galatidis ${ }^{2}$ \\ Maria Drogouti ${ }^{3}$ \\ Iliana Sofianou ${ }^{3}$ \\ Alexis Michail $^{3}$ \\ losif Chatzis ${ }^{3}$ \\ Georgios Donas'
}

'Anesthesiology Department, Agios Dimitrios Hospital,

Thessaloniki, Greece; 'Department of Anesthesiology Nursing, Agios Dimitrios Hospital, Thessaloniki, Greece; ${ }^{3}$ Department of Surgery, Agios Dimitrios Hospital, Thessaloniki, Greece
Correspondence: Dimosthenis Petsas Anesthesiology Department, General Hospital "Agios Dimitrios", Elenis Zografou 2, 54634, Thessaloniki, Greece Tel: +30 23। 3322235

Email dpets_anesthesia@hotmail.com
This article was published in the following Dove Press journal: Journal of Pain Research

\begin{abstract}
The Erector Spinae Plane Block (ESP) is a novel regional technique for anesthesia or analgesia. Originally the ESP block was described in 2016 in a case report regarding analgesia intervention for a case of thoracic neuropathic pain. Since then, there has been growing interest and research adding experience about the ESP block as regional anesthetic and analgesic technique. Reviewing the literature about this novel technique in databases like PubMed using the key words "erector spinae plane block" returns approximately 56 publications. So far there is no available big series of cases or reviews regarding the ESP block. The literature is limited to case reports or case series. With the present case we are interested in exploring the efficacy of ESP block as a postoperative analgesic method for laparoscopic cholecystectomy. We describe the case of a 76-year-old female patient scheduled for laparoscopic cholecystectomy. Written informed consent was granted (for procedure and publication of photos). We applied a bilateral ultrasound-guided ESP block at $\mathrm{T}_{6}$ level while the patient was awake before general anesthesia induction. The anesthetic solution we used consisted of $12 \mathrm{~mL}$ Ropivacaine $0.375 \%$ plus $2 \mathrm{mg}$ dexamethasone (on each side). After the successful administration of the block (observation of the solution spread between transverse process and the erector spinae muscles), general anesthesia was induced and the procedure was started. Procedure and recovery was uneventful with the patient experiencing very good analgesia (NRS pain score 0 up to 6 hours after block placement). The patient presented mild pain (NRS score of 2-3) after 6 hours and requested the "on demand" pain medication ( 1 g paracetamol IV) only 10 hours after the ESP block (NRS pain score of 4-5). The patient experienced no nausea or vomiting, was mobilized easily about 6 hours after the block and was discharged the next day. This relatively simple and safe block dramatically reduced the amount of IV pain medication we usually administer for the specific procedure. The overall result was increased satisfaction of the patient and avoidance of opioid use.
\end{abstract}

Keywords: postoperative analgesia, plane block, ultrasound

\section{Introduction}

Laparoscopic cholecystectomy is one of the most commonly performed procedures in general surgery and it may cause significant postoperative pain and discomfort to the patient. The analgesic regime for postoperative pain usually includes paracetamol, NSAIDs and opioids. ${ }^{1}$ The opioid epidemic as well as the opioid side effects ${ }^{2}$ (sedation, respiratory depression, constipation, delayed patient mobilization) has led perioperative physicians to find a way of decreasing the use of opioids. Increasing the use of regional anesthesia is one of the measures to this end. 
Erector spinae plane (ESP) block is a relatively new technique used for anesthesia or analgesia. The technique was originally described by Forero et $\mathrm{al}^{3}$ in 2016, when it was used to treat thoracic neuropathic pain.

The erector spinae block is achieved by injecting the local anesthetic solution (with possible adjuvants) between the erector spinae muscles (iliocostalis, longissimus, spinalis/ from lateral to medial) and the transverse process (Figure 1). The technique is performed under ultrasound guidance. $A$ high frequency linear probe is placed on a sagittal orientation scanning and identifying the transverse process at the desired level of the vertebral column. The needle is placed in an "in plane" direction. Practically the needle tip is making gentle contact with the posterior surface of the transverse process. Injecting the local anesthetic solution should ideally create an anechoic space between the transverse process and the erector spinae muscles. The local anesthetic is spreading in a caudal and cephalic direction. The solution is probably crossing the internal intercostal membrane blocking dorsal and ventral rami of spinal nerves. The local anesthetic solution is blocking both somatic and sympathetic nerves. ${ }^{4}$

Following the first publication and description of the ESP block there has been a vivid interest in this technique mainly for postoperative analgesia. It combines some favorable characteristics. Simplicity to perform, comparative safety of the technique to paravertebral blocks (aiming on bone structure and not towards paravertebral space near the pleura) effective analgesia and spread on several neurotomes. ${ }^{5}$

Concerning abdominal surgery, it possesses some advantages compared to Transverse Abdominis Plane (TAP) block. TAP block usually achieves a dermatomal block below $\mathrm{T}_{7}$ whereas the ESP block can cover any level. ${ }^{6}$

The ESP block can be performed either as a single shot or as a continuous catheter technique. A great advantage of the ESP block appears to be the provision of both somatic and visceral analgesia. ${ }^{?}$

Research in big databases like PubMed using keywords like "erector spinae plane block" returns a relatively limited number of publications (approximately 57) from which the majority are case reports and case series of this novel technique. The limited literature stresses the usefulness of case reports to increase the volume of knowledge regarding the ESP block. This volume of publications will later be used to create the evidence.

\section{Material and methods}

We describe the case of a 76-year-old, $86 \mathrm{~kg}$ body weight patient scheduled for elective laparoscopic cholecystectomy.
This case report was approved for publication by the local ethics committee according to the principles of the Declaration of Helsinki on ethical principles of medical research. The patient signed informed consent for the case details and images to be published.

The patient had a history of well controlled hypertension under a combination of beta blocker/diuretic and angiotensin II receptor antagonist, hyperlipidemia under ezetimibe/ simvastatin and medication for ulcerative colitis. She had no known allergies. The surgical history included tonsillectomy during childhood. The patient had gastritis untreated and hiatus hernia without reflux symptoms. She was not a smoker and did not drink alcohol.

The chest X-ray showed borderline cardiothoracic ratio with normal lung parenchyma appearance.

Full blood count, biochemistry and coagulation were all within normal limits. The ECG showed signs of left ventricular hypertrophy, but the patient had no clinical signs of cardiac failure (no edema, no paroxysmal nocturnal dyspnea, no orthopnea) and she had a good exercise tolerance (>4 METs). Cardiac echo showed concentric hypertrophy a finding probably related to the patient's hypertension, with good ejection fraction of the left ventricle.

The physical examination was unremarkable.

Benefits and risks of the ESP block was explained in detail to the patient and she signed a written informed consent to undergo a bilateral ESP block while awake before general anesthesia induction.

On the day of the operation the patient was given a mild premedication for anxiolysis. When the patient arrived in the operating room an $18 \mathrm{G}$ cannula was inserted, and an IV fluid drip started. All basic monitoring was applied (ECG/ $\mathrm{HR} / \mathrm{SpO}_{2} / \mathrm{NIBP}$ ), and baseline values documented.

A preliminary scan was done to define and mark the required level $\left(\mathrm{T}_{6}-\mathrm{T}_{7}\right)$, the midline (spinous processes) and bilaterally mark the injection points $3 \mathrm{~cm}$ from midline. The patient was in a sitting position with support from a member of staff (Figure 2). A scan started from lateral to medial in sagittal view. Preparation of the field with iodine was made. The ultrasound anatomical landmarks were identified. The ultrasound anatomical landmarks included the transverse process at $T_{6}$ level and the three layers of muscles from posterior to anterior: trapezius-rhomboiderector spinae (Figures 2 and 3). An $80 \mathrm{~mm} 22 \mathrm{G}$ needle was inserted under ultrasound guidance in plane, aiming towards the transverse process. Gentle contact was made with the transverse process. A total of $12 \mathrm{~mL}$ ropivacaine $0.375 \%$ plus $2 \mathrm{mg}$ dexamethasone was administered on 


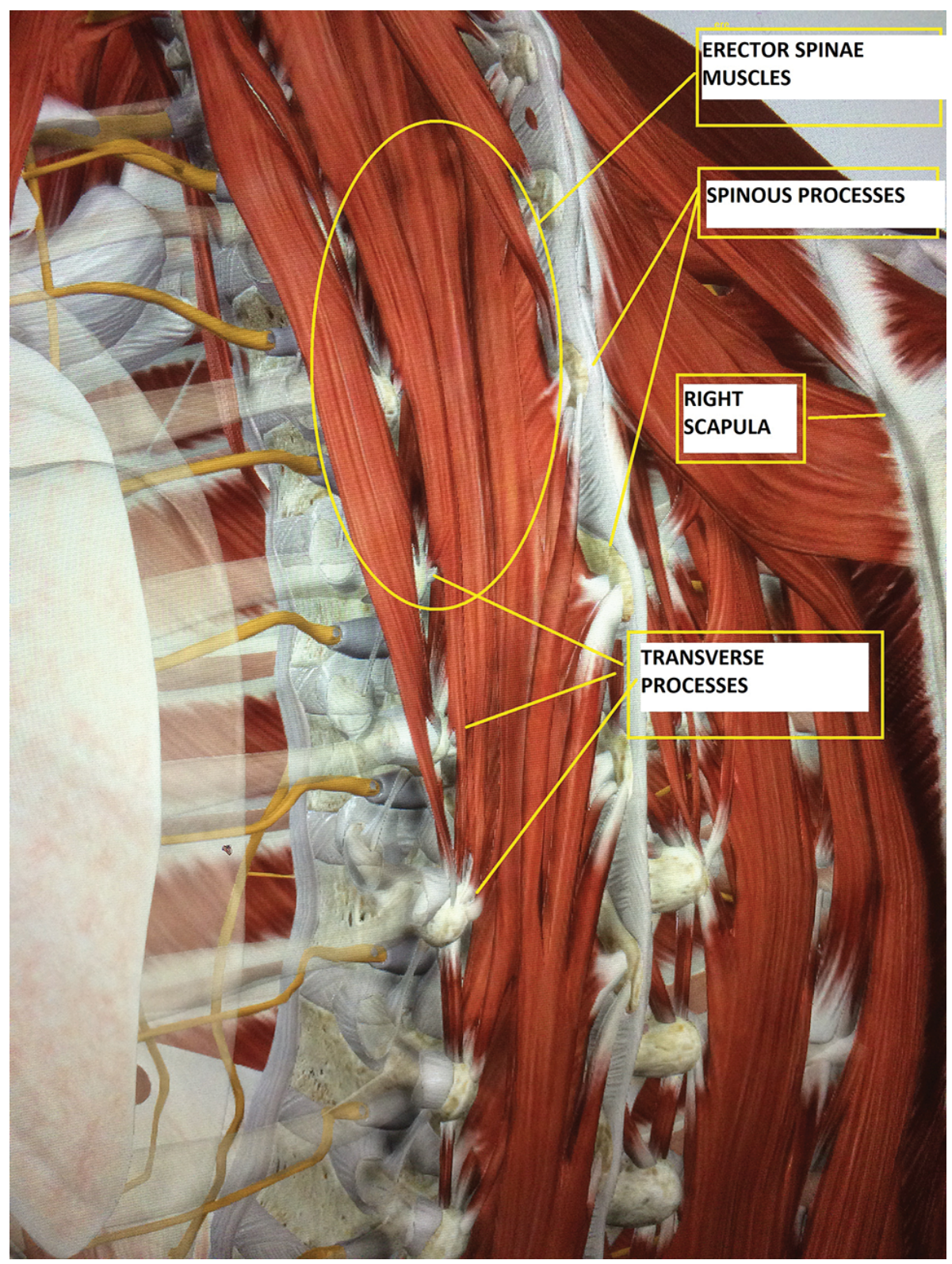

Figure I Anatomy of Erector Spine Muscles area.

each side. The local anesthetic plus the dexamethasone was given in increments with careful repetitive aspiration to avoid intravascular injection.

All necessary precautions for safe administration of the local anesthetics were taken (recurrent aspiration, good needle visualization, feedback about pressure during injection). A good spread anterior to erector spinae muscles was noted (dividing erector spinae muscles from transverse process with a good caudal and cephalic spread).
Following the ESP block, general anesthesia was induced with $150 \mathrm{mg}$ propofol, $75 \mu \mathrm{g}$ fentanyl and $60 \mathrm{mg}$ rocuronium. To avoid any prolonged effect from opioid medication we started an infusion of remifentanil $(0.15 \mu \mathrm{g} / \mathrm{kg} / \mathrm{min})$ and titrated to effect. The choice of remifentanil was based on the fact that the context-sensitive half-life time is short and independent from the duration of infusion. Twenty minutes after the start of the procedure the patient's blood pressure progressively rose, so we titrated remifentanil up to $0.25 \mu \mathrm{g} / \mathrm{kg} /$ 


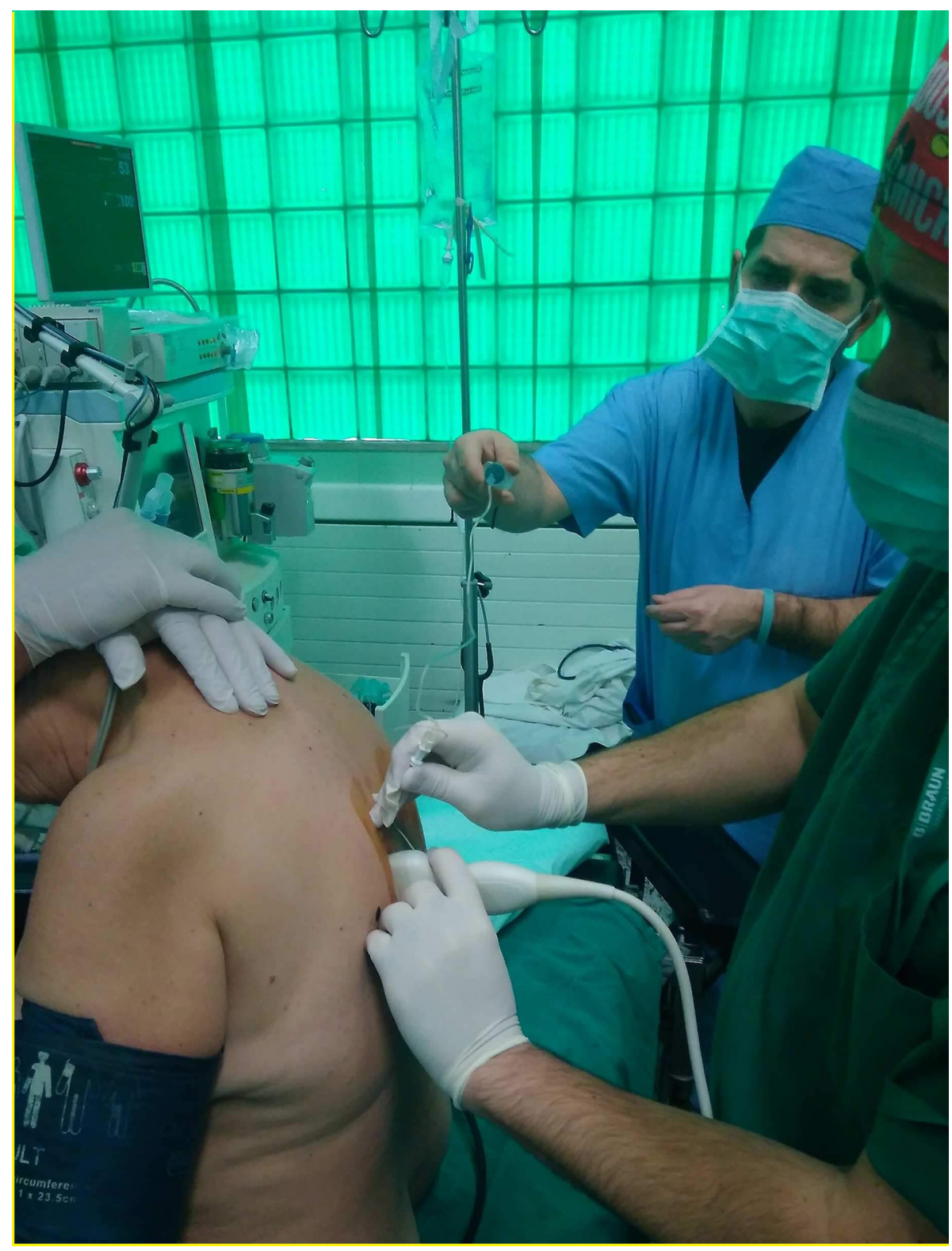

Figure 2 Positioning and scanning during ESP block.

Abbreviation: ESP, Erector Spinae Plane.

min. This fact may be explained by the fact that the ESP block needs about 30 minutes to achieve maximal analgesic effect. Forty minutes after the operation started, we slowly reduced the infusion rate of remifentanil while carefully monitoring vital signs implying pain. Approximately 10 minutes before finishing the procedure, remifentanil was discontinued, and the patient was changed to pressure support ventilation. The patient did not exhibit signs of pain (stable, normal blood pressure, normal to low respiratory rate). The procedure lasted a total of 1 hour and 25 minutes due to anatomical surgical difficulties. Our plan regarding rescue postoperative analgesia included administration of a small IV dose of fentanyl $(25 \mu \mathrm{g}$ IV every 10 minutes up to a maximum of $100 \mu \mathrm{g}$ ) in case the patient complained of pain immediately after recovering from anesthesia. Based on the existing literature we believed that the lower (umbilical) trocar incision would not be covered by the block so we requested the surgeons to infiltrate the area with $5 \mathrm{~mL}$ of $0.5 \%$ ropivacaine.

The patient's pain was monitored using the Numerical Rating Score for pain at times 0-30 minutes, 1, 2, 4, 6, $8,10,12$, and 18 hours. The "zero" point of time was the moment the patient recovered from general anesthesia. 


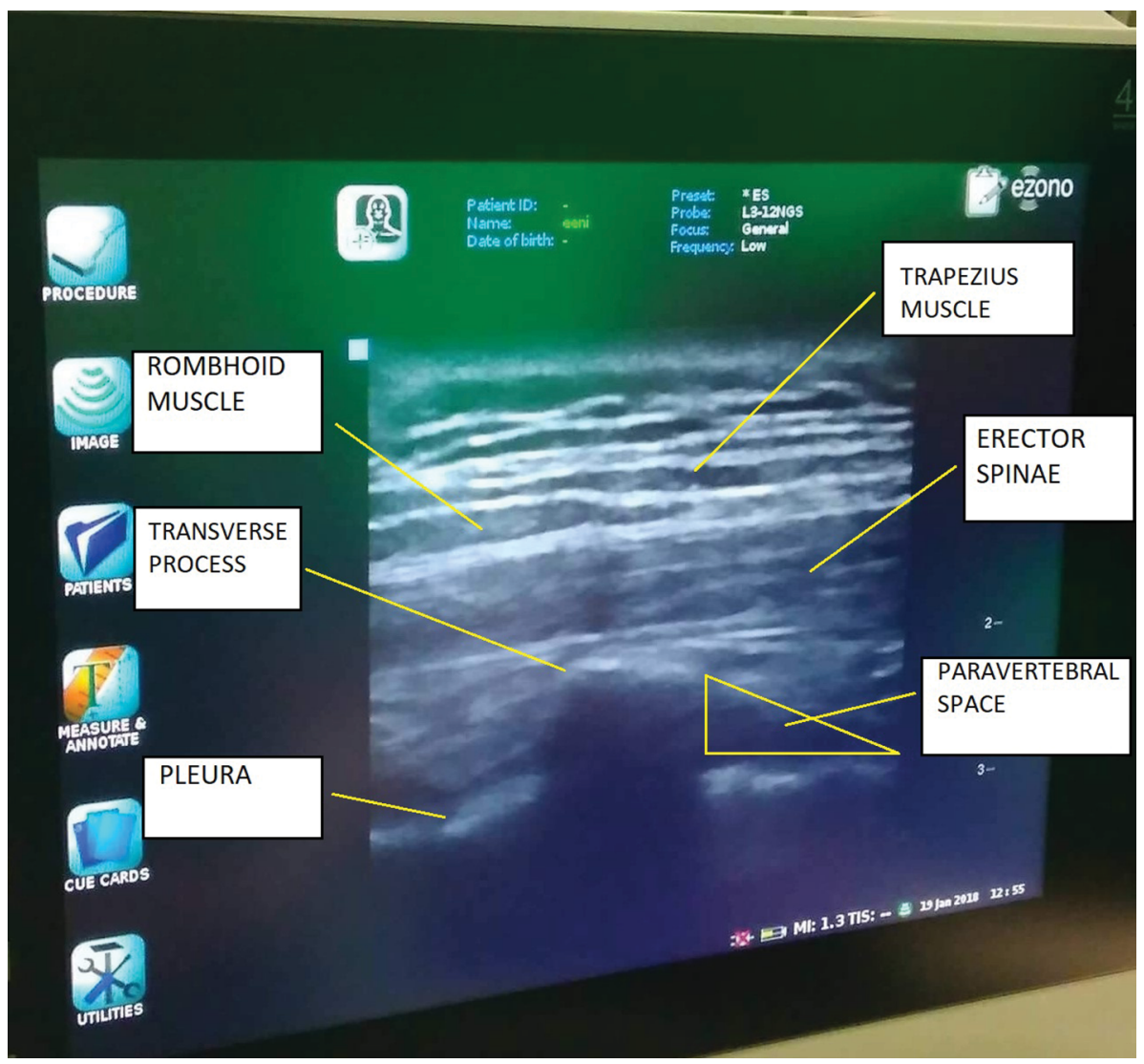

Figure 3 Sonoanatomy during ESP block. Abbreviation: ESP, Erector Spinae Plane.

We prescribed an order for "rescue" medication in case the patient was in pain. This order was all medication on demand and included $1 \mathrm{~g}$ paracetamol IV up to every 6 hours, and if not enough $100 \mathrm{mg}$ tramadol IV with maximum dose every 6 hours.

\section{Results}

Patient recovery was uneventful. When the patient was able to communicate she was totally pain free (NRS $=0$ ).

In the post-anesthesia care unit, using a cold test, patient had a sensory block extending approximately from $\mathrm{T}_{4}-\mathrm{T}_{9}$ which can be seen as the marked area on the patient (Figure 4).

From recovery up to 6 hours after the ESP block the patient was totally pain free. Six hours after the nerve block the patient mentioned having a NRS pain score of 2-3 on deep breath or movement but desired no pain medication. The first dose of on demand pain medication was given 10 hours after the ESP block when the patient had a pain score of NRS 4-5 (1 g paracetamol IV). The patient had no episodes of nausea or vomiting, no problems with her bowel, was mobilized 4-5 hours after the ESP block and was discharged the next morning. Summarizing, except for the ESP block, the only pain medication received by the patient in the perioperative period was $1 \mathrm{~g}$ paracetamol IV. The patient received a prescription for oral analgesics to take home.

\section{Discussion}

The ESP block has a relatively limited literature. In PubMed there are, to date, 57 publications from which 10 papers are technical reports and comments. ${ }^{8-17}$ Thirty publications address thoracic pain or thoracic region surgery management, ${ }^{14-45}$ two papers are on urology, ${ }^{46,47}$ and two papers are on orthopedics. ${ }^{48}$ There are approximately eight publications regarding various applications of ESP or related issues.

The application of ESP block as a technique for analgesia in abdominal surgery is relatively limited and appears in only five publications. This is the reason we believe every new case 


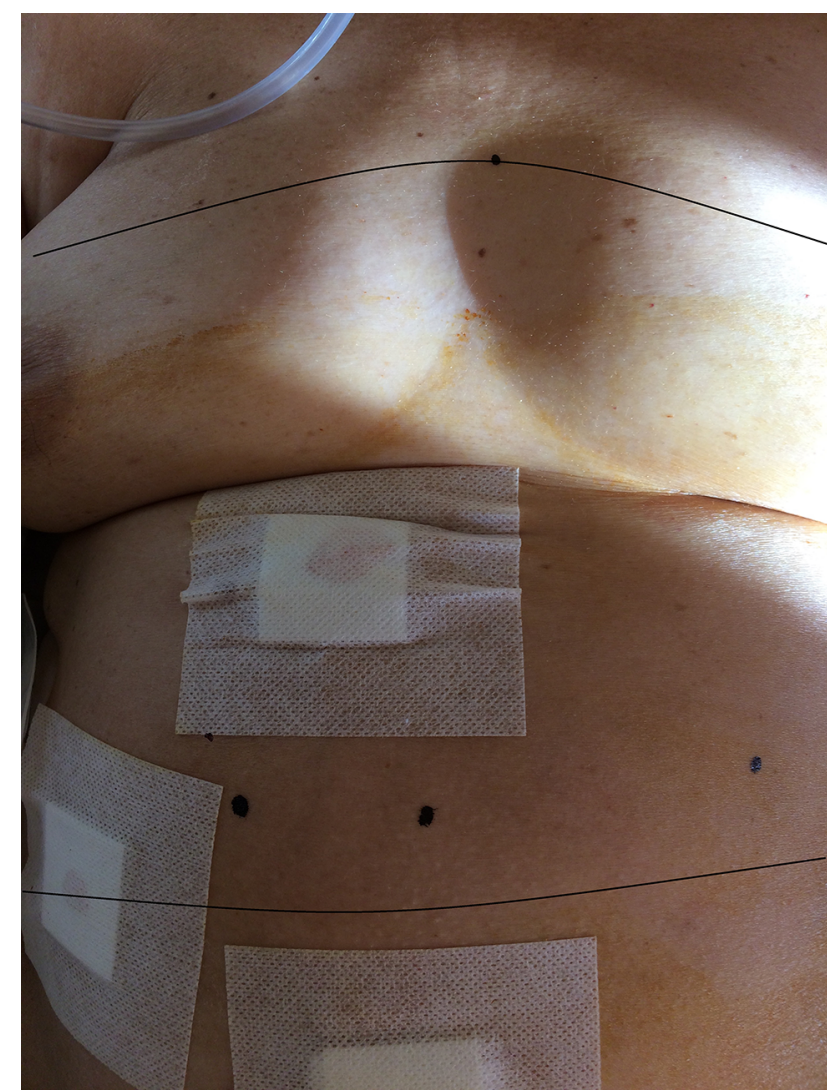

Figure 4 Sensory block area postoperatively (based on cold test).

of ESP block application for abdominal surgery analgesia is a valuable contribution.

In the majority of publications regarding the ESP block the mean volume of local anesthetic solution ranges from 15 to 20 $\mathrm{mL}$ and usually researchers are not using adjuvants to improve the quality or duration of the block. In a few publications there is multimodal analgesia administered regularly which can interfere with the conclusions about ESP block efficacy. Our effort focused on excluding influence of long-acting analgesics on postoperative analgesia. Intraoperatively we administered $75 \mu \mathrm{g}$ fentanyl during induction. Remembering the pharmacokinetics of fentanyl we know that for a given dose around 1 $\mu \mathrm{g} / \mathrm{kg} \mathrm{BW}$ the duration of action is around 20 minutes and in our case our procedure lasted 85 minutes. ${ }^{49}$ Intraoperatively we chose to have an infusion of remifentanil. The first reason was to provide analgesia until the regional block reached peak of action (around 30 minutes). The basic reason for choosing remifentanil was because of its favorable pharmacokinetics. Remifentanil context sensitive half time is short (approximately 3 minutes) and independent of infusion duration. ${ }^{49}$
When we discontinued remifentanil infusion we ensured it would not interfere with postoperative analgesia and conclusions about ESP block efficacy.

The ESP block is a relatively easy to perform, safe technique compared to paravertebral or thoracic epidurals, providing an analgesic effect on desired neurotomes. It significantly seems to reduce the use of opioids and patients can mobilize safely. The conclusion that it seems to reduce opioid use is of course not evidence-based in our case but a result of expert judgement by comparing the usual analgesia demands for the specific procedure. Our findings seem to be in accordance with the very few reports in the literature. Another interesting feedback came from surgical ward nursing, commenting on the minimized time needed to spend with the patient. Of course, this is only an opinion.

\section{Conclusion}

The ESP block presents as a satisfactory analgesic technique providing efficient analgesia in this case of laparoscopic cholecystectomy. The value of adjuvants that prolongs analgesia (as dexamethasone in our case) has been shown in other publications. ${ }^{50}$ There is limited evidence about adjuvants use in the ESP block. Erector spinae plane block is not time consuming and does not greatly affect turnover time in operating theaters. It has the potential of reducing opioid consumption (compared with our usual practice, although one case is insufficient to prove such a hypothesis). Reviewing the literature, the application of ESP block appears to be very promising in reducing opioid-related side effects (like nausea, vomiting, sedation, constipation) and enhancing recovery.

Tis specific case led to an overall satisfactory perioperative experience for patient and family as well as the surgical and anesthesia teams and nursing staff.

Although this case is encouraging we are continuing to perform ESP block in more cases in order to be able to make some statistical comparisons. Overall, we estimate the value of this case report is to add experience about a novel technique with relatively limited literature. The value of case reports in new techniques is to create a volume of knowledge.

\section{Acknowledgments}

We would like to thank Mr Vasilios Skalkos for all the technical support.

We would like to thank the nurses of the Surgery Department of "Agios Dimitrios Hospital" for their valuable feedback and opinion. 


\section{Disclosure}

The authors report no conflicts of interest in this work.

\section{References}

1. Bisgaard T. Analgesic treatment after laparoscopic cholecystectomy: a critical assessment of the evidence. Anesthesiology. 2006;104(4):835-846.

2. Benyamin R, Trescot AM, Etal DS. Opioid complications and side effects. Pain Physician. 2008;11(2 Suppl):105-120.

3. Forero M, Adhikary SD, Lopez H, Tsui C, Chin KJ. The erector spinae plane block: a novel analgesic technique in thoracic neuropathic pain Reg Anesth Pain Med. 2016;41(5):621-627.

4. Ueshima H, Hiroshi O. Spread of local anesthetic solution in the erector spinae plane block. J Clin Anesth. 2018;45:23.

5. El-Boghdadly K, Pawa A. The erector spinae plane block: plane and simple. Anaesthesia. 2017;72(4):434-438.

6. Yarwood J, Berrill A. Nerve blocks of the anterior abdominal wall. Contin Educat Anaesth Crit Care Pain. 2010;10(6):182-186.

7. Chin KJ, Malhas L, Perlas A. The erector spinae plane block provides visceral abdominal analgesia in bariatric surgery: a report of 3 cases. Reg Anesth Pain Med. 2017;42(3):372-376.

8. Josh Luftig PA, Mantuani D, Herring AA, et al. The authors reply to the optimal dose and volume of local anesthetic for erector spinae plane blockade for posterior rib fractures. Am J Emerg Med. 2018;36(6):1103-1104.

9. Bonvicini D, Tagliapietra L. Reply to Dr Casai, et al. J Clin Anesth. 2018;8180(18):30283-30286pii:S0952.

10. de Cassai A, Stefani G, Ori C. Erector spinae plane block and brachial plexus. J Clin Anesth. 2018;45:32.

11. Ueshima H, Hiroshi O. Spread of local anesthetic solution in the erector spinae plane block. J Clin Anesth. 2018;45:23.

12. Costache I, de Neumann L, Ramnanan CJ, et al. The mid-point transverse process to pleura (MTP) block: a new end-point for thoracic paravertebral block. Anaesthesia. 2017;72(10):1230-1236.

13. Fusco P, di Carlo S, Scimia P, et al. Could the new ultrasoundguided erector spinae plane block be a valid alternative to paravertebral block in chronic chest pain syndromes? Minerva Anestesiol. 2017;83(10):1112-1113.

14. Chin KJ, Adhikary S, Forero M. Is the erector spinae plane (ESP) block a sheath block? A reply. Anaesthesia. 2017;72(7):916-917.

15. Hamilton DL, Manickam BP. Is the erector spinae plane (ESP) block a sheath block? Anaesthesia. 2017;72(7):915-916.

16. Murouchi T. Consideration of block nomenclature: erector spinae plane block or retrolaminar block? Reg Anesth Pain Med. 2017;42(1):124.

17. Ueshima H, Otake H. Similarities between the retrolaminar and erector spinae plane blocks. Reg Anesth Pain Med. 2017;42(1):123-124.

18. Kashani HH, Grocott HP. Clarity needed as to the optimal dose and volume of local anesthetic for erector spinae plane blockade for posterior rib fractures. Am J Emerg Med. 2018;36(6):1102-1103.

19. Balaban O, Aydin T, Yaman M. Is ultrasound guided erector spinae plane block sufficient for surgical anesthesia in minor surgery at thoracal region? J Clin Anesth. 2018;47:7-8.

20. Rao Kadam V, Currie J. Ultrasound-guided continuous erector spinae plane block for postoperative analgesia in video-assisted thoracotomy. Anaesth Intensive Care. 2018;46(2):243-245.

21. Singh S, Chowdhary NK. Erector spinae plane block an effective block for post-operative analgesia in modified radical mastectomy. Indian $J$ Anaesth. 2018;62(2):148-150.

22. Nandhakumar A, Nair A, Bharath VK, et al. Erector spinae plane block may aid weaning from mechanical ventilation in patients with multiple rib fractures: case report of two cases. Indian JAnaesth. 2018;62(2):139-141.

23. Ramos J, Peng P, Forero M. Long-term continuous erector spinae plane block for palliative pain control in a patient with pleural mesothelioma. Can J Anaesth. 2018;65(7):852-853.
24. Cesur S, Ay AN, Yayık AM, Naldan ME, Gürkan Y. Ultrasound-guided erector spinae plane block provides effective perioperative analgesia and anaesthesia for thoracic mass excision: a report of two cases. Anaesth Crit Care Pain Med. 2018;5568(17):S235230372-30377pii.

25. Adhikary SD, Pruett A, Forero M, Thiruvenkatarajan V. Erector spinae plane block as an alternative to epidural analgesia for post-operative analgesia following video-assisted thoracoscopic surgery: a case study and a literature review on the spread of local anaesthetic in the erector spinae plane. Indian J Anaesth. 2018;62(1):75-78.

26. Ueshima $\mathrm{H}$, Hiroshi $\mathrm{O}$. Transapical transcatheter aortic valve implantation performed with an erector spinae plane block. J Clin Anesth. 2018;46:84

27. Aydin T, Balaban O, Acar A. Ultrasound guided continuous erector spinae plane block for pain management in pulmonary malignancy. J Clin Anesth. 2018;46:63-64.

28. Gaio-Lima C, Costa CC, Moreira JB, Lemos TS, Trindade HL. Continuous erector spinae plane block for analgesia in pediatric thoracic surgery: a case report. Rev Esp Anestesiol Reanim. 2018;65(5):2873026930290pii.

29. Luis-Navarro JC, Seda-Guzmán M, Luis-Moreno C, López-Romero JL. The erector spinae plane block in 4 cases of video-assisted thoracic surgery. Rev Esp Anestesiol Reanim. 2018;65(4):204-208.

30. Luftig J, Mantuani D, Herring AA, Dixon B, Clattenburg E, Nagdev A. Successful emergency pain control for posterior rib fractures with ultrasound-guided erector spinae plane block. Am J Emerg Med. 2018;36(8):1391-1396.

31. Hernandez MA, Palazzi L, Lapalma J, Forero M, Chin KJ. Erector spinae plane block for surgery of the posterior thoracic wall in a pediatric patient. Reg Anesth Pain Med. 2018;43(2):217-219.

32. Ahiskalioglu A, Alici HA, Ari MA. Ultrasound guided low thoracic erector spinae plane block for management of acute herpes zoster. $J$ Clin Anesth. 2018;45:60-61.

33. Tanaka N, Ueshima H, Otake H. Erector spinae plane block for combined lovectomy and radical mastectomys. J Clin Anesth. 2018;45:27-28.

34. Kumar A, Hulsey A, Martinez-Wilson H. The use of liposomal bupivacaine in erector spinae plane block to minimize opioid consumption for breast surgery: a case report. A A Pract. 2018;10(9):239-241.

35. Tulgar S, Senturk O. Ultrasound guided erector spinae plane block at L-4 transverse process level provides effective postoperative analgesia for total hip arthroplasty. J Clin Anesth. 2018;44:68.

36. Bonvicini D, Tagliapietra L, Giacomazzi A, Pizzirani E. Bilateral ultrasound-guided erector spinae plane blocks in breast cancer and reconstruction surgery. J Clin Anesth. 2018;44:3-4.

37. Ohgoshi Y, Ikeda T, Kurahashi K. Continuous erector spinae plane block provides effective perioperative analgesia for breast reconstruction using tissue expanders: a report of two cases. J Clin Anesth. 2018;44:1-2.

38. Forero M, Rajarathinam M, Adhikary S, Chin KJ. Erector spinae plane (ESP) block in the management of post thoracotomy pain syndrome: a case series. Scand J Pain. 2017;17:325-329.

39. Scimia P, Basso Ricci E, Droghetti A, Fusco P. The ultrasound-guided continuous erector spinae plane block for postoperative analgesia in video-assisted thoracoscopic lobectomy. Reg Anesth Pain Med. 2017;42(4):537.

40. Ueshima H, Otake H. Erector spinae plane block provides effective pain management during pneumothorax surgery. JClin Anesth. 2017;40:74.

41. Bonvicini D, Giacomazzi A, Pizzirani E. Use of the ultrasound-guided erector spinae plane block in breast surgery. Minerva Anestesiol. 2017;83(10):1111-1112.

42. Muñoz F, Cubillos J, Bonilla AJ, Chin KJ. Erector spinae plane block for postoperative analgesia in pediatric oncological thoracic surgery. Can J Anaesth. 2017;64(8):880-882.

43. Ueshima H, Otake H. Clinical experiences of ultrasound-guided erector spinae plane block for thoracic vertebra surgery. J Clin Anesth. 2017;38:137.

44. Forero M, Rajarathinam M, Adhikary S, Chin KJ. Continuous erector spinae plane block for rescue analgesia in thoracotomy after epidural failure: a case report. A A Case Rep. 2017;8(10):254-256. 
45. Hamilton DL, Manickam B. Erector spinae plane block for pain relief in rib fractures. Br J Anaesth. 2017;118(3):474-475.

46. Munshey F, Rodriguez S, Diaz E, Tsui B. Continuous erector spinae plane block for an open pyeloplasty in an infant. J Clin Anesth. 2018;47(21):47-49.

47. Tulgar S, Senturk O. Ultrasound guided low thoracic erector spinae plane block for postoperative analgesia in radical retropubic prostatectomy, a new indication. $J$ Clin Anesth. 2018;47:4-4.
48. Tulgar S, Selvi O, Senturk O, et al. Clinical experiences of ultrasoundguided lumbar erector spinae plane block for hip joint and proximal femur surgeries. J Clin Anesth. 2018;47:5-6.

49. Yentis S, Hirsch N, Ip J. Anaesthesia and Intensive Care A-Z. 5th ed. London: Churchill Livingstone; 2013.

50. Choi S, Rodseth R, Mccartney CJ. Effects of dexamethasone as a local anaesthetic adjuvant for brachial plexus block: a systematic review and meta-analysis of randomized trials. Br JAnaesth. 2014;112(3):427-439.

\section{Publish your work in this journal}

The Journal of Pain Research is an international, peer reviewed, open access, online journal that welcomes laboratory and clinical findings in the fields of pain research and the prevention and management of pain. Original research, reviews, symposium reports, hypothesis formation and commentaries are all considered for publication.
Dovepress

The manuscript management system is completely online and includes a very quick and fair peer-review system, which is all easy to use. Visit http://www.dovepress.com/testimonials.php to read real quotes from published authors. 